\title{
ON THE LEBEDEV TRANSFORMATION IN HARDY'S SPACES
}

\author{
SEMYON B. YAKUBOVICH
}

Received 31 January 2003

\begin{abstract}
We establish the inverse Lebedev expansion with respect to parameters and arguments of the modified Bessel functions for an arbitrary function from Hardy's space $H_{2, A}, A>0$. This gives another version of the Fourier-integral-type theorem for the Lebedev transform. The result is generalized for a weighted Hardy space $\hat{H}_{2, A} \equiv \hat{H}_{2}\left((-A, A) ;|\Gamma(1+\operatorname{Re} z+i \tau)|^{2} d \tau\right)$, $0<A<1$, of analytic functions $f(z), z=\operatorname{Re} z+i \tau$, in the strip $|\operatorname{Re} z| \leq A$. Boundedness and inversion properties of the Lebedev transformation from this space into the space $L_{2}\left(\mathbb{R}_{+} ; x^{-1} d x\right)$ are considered. When $\operatorname{Re} z=0$, we derive the familiar Plancherel theorem for the Kontorovich-Lebedev transform.
\end{abstract}

2000 Mathematics Subject Classification: 44A15, 42B30, 33C10.

1. Introduction. In 1947 Lebedev [4] proved the following expansion of an arbitrary function $g$ in terms of the modified Bessel functions

$$
g(x)=\frac{1}{\pi i} \int_{\alpha-i \infty}^{\alpha+i \infty} \mu K_{\mu}(x) d \mu \int_{0}^{\infty} \frac{g(t)}{t} I_{\mu}(t) d t, \quad x>0
$$

Here $K_{\mu}(x), I_{\mu}(x)$ are modified Bessel functions [2, 5] of the complex index $\mu=\alpha+i \tau$, $\alpha>1, x>0, g$ is an arbitrary function of bounded variation in any finite interval and it belongs to the weighted Lebesgue space $L_{1}\left(\mathbb{R}_{+} ; x^{-1} I_{\alpha}(x) d x\right)$. The outer integral in (1.1) is understood, as usual, in a principal value sense.

Our object here is to study the inverse Lebedev expansion

$$
f(z)=\frac{1}{\pi i} \int_{0}^{\infty} \frac{I_{z}(t)}{t} d t \int_{\alpha-i \infty}^{\alpha+i \infty} \mu K_{\mu}(t) f(\mu) d \mu, \quad \operatorname{Re} z>|\alpha|,
$$

in the Hardy space $H_{2, A}, A>0[3,6]$, which consists of those functions $f(z), z=\operatorname{Re} z+$ $i \tau$, analytic in the right half-plane $\operatorname{Re} z>-A$ with the property

$$
\|f\|_{H_{2, A}}=\sup _{\operatorname{Re} z>-A}\left(\int_{-\infty}^{\infty}|f(\operatorname{Re} z+i \tau)|^{2} d \tau\right)^{1 / 2}<\infty .
$$

Expansion (1.2) generates the pair of Lebedev integral transformations

$$
\begin{gathered}
g(x)=\frac{1}{\pi i} \int_{\alpha-i \infty}^{\alpha+i \infty} \mu K_{\mu}(x) f(\mu) d \mu, \\
f(z)=\int_{0}^{\infty} \frac{I_{z}(t)}{t} g(t) d t,
\end{gathered}
$$


with respect to an index and an argument of the modified Bessel functions. For $0<A<1$, we extend Lebedev's transformation on the generalized Hardy space

$$
\hat{H}_{2, A} \equiv \hat{H}_{2}\left((-A, A) ;|\Gamma(1+\operatorname{Re} z+i \tau)|^{2} d \tau\right) \supset H_{2, A}
$$

of analytic functions $f(z)$ in the strip $|\operatorname{Re} z| \leq A$ with the condition

$$
\|f\|_{\hat{H}_{2, A}}=\sup _{|\operatorname{Re} z| \leq A}\left(\int_{-\infty}^{\infty}|\Gamma(1+\operatorname{Re} z+i \tau)|^{2}|f(\operatorname{Re} z+i \tau)|^{2} d \tau\right)^{1 / 2}<\infty,
$$

where $\Gamma(z)$ is Euler's gamma function. The boundedness theorems of Plancherel type are proved in these spaces. In particular, the case $\operatorname{Re} z=0$ in (1.7) leads to the Plancherel theorem for the familiar Kontorovich-Lebedev transform (cf. [9, Chapter 2]).

We note (see $[2,7,9])$ that the modified Bessel functions $K_{\mu}(z), I_{\mu}(z)$ are linear independent solutions of the Bessel differential equation

$$
z^{2} \frac{d^{2} u}{d z^{2}}+z \frac{d u}{d z}-\left(z^{2}+\mu^{2}\right) u=0
$$

They can be given by the formulas

$$
\begin{gathered}
I_{\mu}(z)=\sum_{k=0}^{\infty} \frac{(z / 2)^{\mu+2 k}}{\Gamma(\mu+k+1) k !}, \\
K_{\mu}(z)=\frac{\pi}{2 \sin \pi \mu}\left[I_{-\mu}(z)-I_{\mu}(z)\right],
\end{gathered}
$$

when $\mu \neq 0, \pm 1, \pm 2, \ldots$, and $K_{n}(z)=\lim _{\mu \rightarrow n} K_{\mu}(z), n=0, \pm 1, \pm 2, \ldots$ The function $K_{\mu}(z)$ is also called the MacDonald function and has the following integral representations (cf. [2, 9]):

$$
K_{\mu}(z)=\int_{0}^{\infty} e^{-z \cosh t} \cosh \mu t d t=\frac{1}{2} \int_{0}^{\infty} e^{-z\left(t+t^{-1}\right) / 2} t^{\mu-1} d t .
$$

Useful relations are [2, 7]

$$
\begin{gathered}
2 \mu K_{\mu}(z)=z\left[K_{\mu+1}(z)-K_{\mu-1}(z)\right], \\
\int_{0}^{\infty} I_{\xi}(x) K_{\mu}(x) \frac{d x}{x}=\frac{1}{\xi^{2}-\mu^{2}}, \quad \operatorname{Re} \xi>|\operatorname{Re} \mu|, \\
\frac{d^{n}}{d z^{n}} K_{\mu}(z)=\frac{(-1)^{n}}{2^{n}} \sum_{k=0}^{n}\left(\begin{array}{l}
n \\
k
\end{array}\right) K_{\mu-n+2 k}(z) .
\end{gathered}
$$

These functions have the asymptotic behavior [2, 5]

$$
\begin{gathered}
K_{\mu}(z)=\left(\frac{\pi}{2 z}\right)^{1 / 2} e^{-z}\left[1+O\left(\frac{1}{z}\right)\right], \quad z \rightarrow \infty, \\
I_{\mu}(z)=\frac{e^{z}}{\sqrt{2 \pi z}}\left[1+O\left(\frac{1}{z}\right)\right], \quad z \longrightarrow \infty,
\end{gathered}
$$


and near the origin

$$
\begin{aligned}
K_{\mu}(z) & =O\left(z^{-|\operatorname{Re} \mu|}\right), \quad z \longrightarrow 0, \\
K_{0}(z) & =-\log z+O(1), \quad z \longrightarrow 0, \\
I_{\mu}(z) & =O\left(z^{\operatorname{Re} \mu}\right), \quad \mu \neq 0, z \longrightarrow 0 .
\end{aligned}
$$

Meanwhile asymptotic formulas (1.15), (1.16), (1.17), (1.18), (1.19) can be written in a more explicit form. In particular, formula (1.15) has the following interpretation (cf. [5, Section 6.2.7, formula (38)]):

$$
\begin{aligned}
K_{\mu}(z) & \sim\left(\frac{\pi}{2 z}\right)^{1 / 2} e^{-z} F_{0}\left(\frac{1}{2}+\mu, \frac{1}{2}-\mu ;-\frac{1}{2 z}\right) \\
& =\left(\frac{\pi}{2 z}\right)^{1 / 2} e^{-z} \sum_{n=0}^{\infty}\left(\frac{1}{2}+\mu\right)_{n}\left(\frac{1}{2}-\mu\right)_{n} \frac{(-1)^{n}}{n !(2 z)^{n}}, \quad z \rightarrow \infty
\end{aligned}
$$

where ${ }_{2} F_{0}(a, b ; z)$ is the generalized hypergeometric function and $(a)_{n}=a(a+1) \ldots$ $(a+n-1)$ is Pochhammer's symbol [1]. We note that according to [2, Chapter 7] the equivalence sign in (1.20) means that for any fixed $\mu$ and for each $M=1,2, \ldots$, we have the exact equality

$$
K_{\mu}(z)=\left(\frac{\pi}{2 z}\right)^{1 / 2} e^{-z}\left[\sum_{n=0}^{M-1}\left(\frac{1}{2}+\mu\right)_{n}\left(\frac{1}{2}-\mu\right)_{n} \frac{(-1)^{n}}{n !(2 z)^{n}}+O\left(|z|^{-M}\right)\right], \quad z \rightarrow \infty .
$$

We will use below the properties of the Mellin transform pair [8], which is defined by the formulas

$$
\begin{gathered}
f^{\mathcal{M}}(s)=\int_{0}^{\infty} f(x) x^{s-1} d x, \\
f(x)=\frac{1}{2 \pi i} \int_{\gamma-i \infty}^{\gamma+i \infty} f^{\mu}(s) x^{-s} d s, \quad s=\gamma+i t, x>0,
\end{gathered}
$$

where integrals (1.22) exist as Lebesgue integrals or, in particular, in mean with respect to the norm of spaces $L_{2}(\gamma-i \infty, \gamma+i \infty)$ and $L_{2}\left(\mathbb{R}_{+} ; x^{2 \gamma-1}\right)$, respectively. In the latter case, the Parseval equality of the form

$$
\int_{0}^{\infty}|f(x)|^{2} x^{2 \gamma-1} d x=\frac{1}{2 \pi} \int_{-\infty}^{\infty}\left|f^{\mu}(\gamma+i t)\right|^{2} d t
$$

holds true.

2. Lebedev's transform in Hardy's spaces. We begin with the following.

THEOREM 2.1. Let $f \in H_{2, A}$. Then expansion (1.2) is true for all $z$ such that $\operatorname{Re} z>|\alpha|$, $\alpha>-A$, where integrals with respect to $\mu$ and $t$ exist in Lebesgue's sense.

Proof. First we show under conditions of the theorem that the inner integral in (1.2) exists as a Lebesgue integral. Then we will obtain an estimate, which will provide 
the absolute and uniform convergence by $\operatorname{Re} z \geq \alpha_{0}>|\alpha|$ of the outer integral in (1.2). In fact, invoking Schwarz's inequality we have

$$
\begin{aligned}
\left|\int_{\alpha-i \infty}^{\alpha+i \infty} \frac{\mu K_{\mu}(t)}{t} f(\mu) d \mu\right| & \leq \int_{-\infty}^{\infty}\left|(\alpha+i \tau) \frac{K_{\alpha+i \tau}(t)}{t} f(\alpha+i \tau)\right| d \tau \\
& \leq\|f\|_{H_{2, A}}\left(\int_{-\infty}^{\infty}\left|(\alpha+i \tau) \frac{K_{\alpha+i \tau}(t)}{t}\right|^{2} d \tau\right)^{1 / 2} .
\end{aligned}
$$

We treat the latter integral by using integral representations (1.11) of the MacDonald function, relation (1.12), and the Parseval equality (1.23) for the Mellin transform. Indeed, we deduce

$$
\begin{aligned}
(\alpha+i \tau) \frac{K_{\alpha+i \tau}(t)}{t} & =\frac{1}{2}\left[K_{1+\alpha+i \tau}(t)-K_{\alpha+i \tau-1}(t)\right] \\
& =\frac{1}{4} \int_{0}^{\infty} e^{-t\left(y+y^{-1}\right) / 2}\left(y-y^{-1}\right) y^{\alpha+i \tau-1} d y .
\end{aligned}
$$

Hence taking into account the reciprocal formulas of the Mellin transform (1.22) via (1.23), and (1.11), we find

$$
\begin{aligned}
\int_{-\infty}^{\infty}\left|(\alpha+i \tau) \frac{K_{\alpha+i \tau}(t)}{t}\right|^{2} d \tau & =\frac{1}{16} \int_{-\infty}^{\infty}\left|\int_{0}^{\infty} e^{-t\left(y+y^{-1}\right) / 2}\left(y-y^{-1}\right) y^{\alpha+i \tau-1} d y\right|^{2} d \tau \\
& =\frac{\pi}{8} \int_{0}^{\infty} e^{-t\left(y+y^{-1}\right)}\left(y-y^{-1}\right)^{2} y^{2 \alpha-1} d y \\
& =\frac{\pi}{4}\left[K_{2(\alpha+1)}(2 t)+K_{2(\alpha-1)}(2 t)-2 K_{2 \alpha}(2 t)\right], \quad \alpha \in \mathbb{R} .
\end{aligned}
$$

Therefore combining with (2.1) we obtain the estimate

$$
\begin{aligned}
\int_{-\infty}^{\infty} & \left|(\alpha+i \tau) \frac{K_{\alpha+i \tau}(t)}{t} f(\alpha+i \tau)\right| d \tau \\
\leq & \frac{\sqrt{\pi}}{2}\|f\|_{H_{2, A}}\left(K_{2(\alpha+1)}(2 t)+K_{2(\alpha-1)}(2 t)-2 K_{2 \alpha}(2 t)\right)^{1 / 2} .
\end{aligned}
$$

Hence by using (2.4) we derive the absolute convergence of the iterated integral (1.2) under condition $\operatorname{Re} z>|\alpha|$. In fact, it will follow from the convergence of the integral

$$
\int_{0}^{\infty}\left|I_{z}(t)\right|\left(K_{2(\alpha+1)}(2 t)+K_{2(\alpha-1)}(2 t)-2 K_{2 \alpha}(2 t)\right)^{1 / 2} d t<\infty,
$$

where $\operatorname{Re} z>|\alpha|$. We apply asymptotic formulas (1.17), (1.18) and expansion (1.21) in terms of the generalized hypergeometric series (see also [5]) for the modified Bessel functions. Now it is not difficult to obtain principal asymptotic terms near the origin and at infinity for the latter combination of the MacDonald functions in (2.5). Indeed, for the case of infinity it is sufficient to put in (1.21) $M=2$. Precisely, we find

$$
\begin{aligned}
& \left(K_{2(\alpha+1)}(2 t)+K_{2(\alpha-1)}(2 t)-2 K_{2 \alpha}(2 t)\right)^{1 / 2}=O\left(t^{-|\alpha|-1}\right), \quad t \rightarrow 0+, \\
& \left(K_{2(\alpha+1)}(2 t)+K_{2(\alpha-1)}(2 t)-2 K_{2 \alpha}(2 t)\right)^{1 / 2}=\frac{\pi^{1 / 4}}{t^{3 / 4}} e^{-t}\left(1+O\left(\frac{1}{t}\right)\right), \quad t \rightarrow+\infty,
\end{aligned}
$$


where the corresponding constant under the sign $O$ is an absolute one. Consequently, invoking asymptotic relations (1.16), (1.19), we easily establish the convergence of the integral (2.5) when $\operatorname{Re} z>|\alpha|$ and its uniform convergence by $\operatorname{Re} z \geq \alpha_{0}>|\alpha|$. Hence by virtue of Fubini's theorem we invert the order of integration in the right-hand side of (1.2). Calculating the integral with respect to $t$ by (1.13), we get the representation

$$
\frac{1}{\pi i} \int_{0}^{\infty} \frac{I_{z}(t)}{t} d t \int_{\alpha-i \infty}^{\alpha+i \infty} \mu K_{\mu}(t) f(\mu) d \mu=\frac{1}{\pi i} \int_{\alpha-i \infty}^{\alpha+i \infty} \frac{\mu f(\mu) d \mu}{z^{2}-\mu^{2}} .
$$

Now in order to complete the proof of Theorem 2.1 we will prove that the latter integral is $f(z)$. For this we use the theory of Cauchy's integrals. We write the right-hand side of (2.7) in the form

$$
\frac{1}{\pi i} \int_{\alpha-i \infty}^{\alpha+i \infty} \frac{\mu f(\mu) d \mu}{z^{2}-\mu^{2}}=\frac{1}{2 \pi i} \int_{\alpha+i \infty}^{\alpha-i \infty} \frac{2 \mu f(\mu) d \mu}{(\mu-z)(\mu+z)} .
$$

However, since $\operatorname{Re} z>|\alpha|$ and $B, C>0$ are large enough, then by Cauchy's theorem we have

$$
f(z)=\frac{1}{2 \pi i}\left[\int_{\alpha+i B}^{\alpha-i B}+\int_{\alpha-i B}^{C-i B}+\int_{C-i B}^{C+i B}+\int_{C+i B}^{\alpha+i B}\right] \frac{2 \mu f(\mu) d \mu}{(\mu-z)(\mu+z)} .
$$

By a further integration,

$$
\begin{aligned}
f(z) & =\frac{1}{2 \pi i} \int_{C}^{C+1} d B\left[\int_{\alpha+i B}^{\alpha-i B}+\int_{\alpha-i B}^{C-i B}+\int_{C-i B}^{C+i B}+\int_{C+i B}^{\alpha+i B}\right] \frac{2 \mu f(\mu) d \mu}{(\mu-z)(\mu+z)} \\
& =J_{1}+J_{2}+J_{3}+J_{4} .
\end{aligned}
$$

Hence we choose $C$ such that $|z|<C / \sqrt{2}$. Then

$$
\begin{aligned}
\left|J_{2}\right| & =\frac{1}{\pi}\left|\int_{C}^{C+1} d B \int_{\alpha-i B}^{C-i B} \frac{\mu f(\mu) d \mu}{\mu^{2}-z^{2}}\right|=\frac{1}{\pi}\left|\int_{\alpha}^{C} d u \int_{C}^{C+1} \frac{(u-i B) f(u-i B) d B}{(u-i B)^{2}-z^{2}}\right| \\
& \leq \frac{1}{\pi} \int_{\alpha}^{C} d u\left(\int_{C}^{C+1}|f(u-i B)|^{2} d B\right)^{1 / 2}\left(\int_{C}^{C+1} \frac{|u-i B|^{2} d B}{\left|(u-i B)^{2}-z^{2}\right|^{2}}\right)^{1 / 2} \\
& \leq \frac{1}{\pi} \sup _{x>-A}\left(\int_{C}^{C+1}|f(x-i B)|^{2} d B\right)^{1 / 2} \int_{\alpha}^{C} d u\left(\int_{C}^{C+1} \frac{|u-i B|^{2} d B}{|| u-\left.i B\right|^{2}-\left.|z|^{2}\right|^{2}}\right)^{1 / 2} .
\end{aligned}
$$

But

$$
\begin{aligned}
& \int_{\alpha}^{C} d u\left(\int_{C}^{C+1} \frac{|u-i B|^{2} d B}{|| u-\left.i B\right|^{2}-\left.|z|^{2}\right|^{2}}\right)^{1 / 2} \\
& \quad \leq \int_{\alpha}^{C} d u\left(\int_{C}^{C+1} \frac{\left(u^{2}+B^{2}\right) d B}{\left(u^{2}+B^{2} / 2\right)^{2}}\right)^{1 / 2}=2 \int_{\alpha}^{C} \frac{d u}{\sqrt{|u|}}\left(\int_{C /|u|}^{(C+1) /|u|} \frac{\left(1+v^{2}\right) d v}{\left(2+v^{2}\right)^{2}}\right)^{1 / 2} \\
& \quad \leq 2 \int_{\alpha}^{C} \sqrt{\frac{1}{|u|} \arctan \frac{|u|}{u^{2}+C(C+1)}} d u \leq 2 \sqrt{\arctan \frac{1}{2 C}} \int_{\alpha}^{C} \frac{d u}{\sqrt{|u|}} \\
& \quad \leq \sqrt{\frac{2}{C}} \int_{-C}^{C} \frac{d u}{\sqrt{|u|}}=4 \sqrt{2} .
\end{aligned}
$$


Consequently we obtain

$$
\left|J_{2}\right| \leq \frac{4 \sqrt{2}}{\pi} \sup _{x>-A}\left(\int_{C}^{C+1}|f(x-i B)|^{2} d B\right)^{1 / 2} \longrightarrow 0, \quad C \rightarrow \infty .
$$

Similarly,

$$
\left|J_{4}\right|=\frac{1}{\pi}\left|\int_{C}^{C+1} d B \int_{C+i B}^{\alpha+i B} \frac{\mu f(\mu) d \mu}{\mu^{2}-z^{2}}\right| \rightarrow 0, \quad C \longrightarrow \infty .
$$

Further,

$$
\begin{aligned}
\left|J_{3}\right| & =\frac{1}{\pi}\left|\int_{C}^{C+1} d B \int_{C-i B}^{C+i B} \frac{\mu f(\mu) d \mu}{\mu^{2}-z^{2}}\right| \leq \frac{1}{\pi} \int_{-\infty}^{\infty} \frac{|C+i \tau||f(C+i \tau)| d \tau}{\left|(C+i \tau)^{2}-z^{2}\right|} \\
& \leq \frac{1}{\pi}\|f\|_{H_{2, A}}\left(\int_{-\infty}^{\infty} \frac{|C+i \tau|^{2} d \tau}{\left|(C+i \tau)^{2}-z^{2}\right|^{2}}\right)^{1 / 2} \leq \frac{1}{\pi}\|f\|_{H_{2, A}}\left(\int_{-\infty}^{\infty} \frac{|C+i \tau|^{2} d \tau}{|| C+\left.i \tau\right|^{2}-C^{2} /\left.2\right|^{2}}\right)^{1 / 2} \\
& =\frac{2}{\pi \sqrt{C}}\|f\|_{H_{2, A}}\left(\int_{-\infty}^{\infty} \frac{\left(1+u^{2}\right) d u}{\left(1+2 u^{2}\right)^{2}}\right)^{1 / 2} \leq \frac{2}{\sqrt{\pi C}}\|f\|_{H_{2, A}} \rightarrow 0, \quad C \rightarrow \infty .
\end{aligned}
$$

Thus

$$
\begin{aligned}
& f(z)= \lim _{C \rightarrow \infty} \frac{1}{\pi i} \int_{C}^{C+1} d B \int_{-B}^{B} \frac{(\alpha+i \tau) f(\alpha+i \tau) d \tau}{z^{2}-(\alpha+i \tau)^{2}} \\
&=\lim _{C \rightarrow \infty}\left[\frac{1}{\pi i} \int_{-C}^{C} \frac{(\alpha+i \tau) f(\alpha+i \tau) d \tau}{z^{2}-(\alpha+i \tau)^{2}}+\frac{1}{\pi i} \int_{-C-1}^{-C} \frac{(C+1+\tau)(\alpha+i \tau) f(\alpha+i \tau) d \tau}{z^{2}-(\alpha+i \tau)^{2}}\right. \\
&\left.\quad+\frac{1}{\pi i} \int_{C}^{C+1} \frac{(C+1-\tau)(\alpha+i \tau) f(\alpha+i \tau) d \tau}{z^{2}-(\alpha+i \tau)^{2}}\right] .
\end{aligned}
$$

However,

$$
\begin{aligned}
& \frac{1}{\pi}\left|\int_{C}^{C+1} \frac{(C+1-\tau)(\alpha+i \tau) f(\alpha+i \tau) d \tau}{z^{2}-(\alpha+i \tau)^{2}}\right| \\
& \quad \leq \frac{1}{\pi}\|f\|_{H_{2, A}}\left(\int_{C}^{C+1} \frac{(C+1-\tau)^{2}|\alpha+i \tau|^{2} d \tau}{\left|z^{2}-(\alpha+i \tau)^{2}\right|^{2}}\right)^{1 / 2} .
\end{aligned}
$$

Further, since $|z|<C / \sqrt{2}$, we have

$$
\begin{aligned}
& \left(\int_{C}^{C+1} \frac{(C+1-\tau)^{2}|\alpha+i \tau|^{2} d \tau}{\left|z^{2}-(\alpha+i \tau)^{2}\right|^{2}}\right)^{1 / 2} \\
& \quad \leq\left(\int_{C}^{C+1} \frac{(C+1-\tau)^{2}\left(\alpha^{2}+\tau^{2}\right) d \tau}{\left(\alpha^{2}+\tau^{2} / 2\right)^{2}}\right)^{1 / 2} \leq 2\left(\int_{C}^{C+1} \frac{(C+1-\tau)^{2} d \tau}{\alpha^{2}+\tau^{2}}\right)^{1 / 2} \\
& \quad=2\left((C+1) \int_{C /(C+1)}^{1} \frac{(1-v)^{2} d v}{\alpha^{2} /(C+1)^{2}+v^{2}}\right)^{1 / 2} \leq \frac{2}{C}
\end{aligned}
$$


Combining with (2.17) we get that the integral in its left-hand side tends to zero, when $C \rightarrow \infty$. In the same manner we derive

$$
\lim _{C \rightarrow \infty} \frac{1}{\pi i} \int_{-C-1}^{-C} \frac{(C+1+\tau)(\alpha+i \tau) f(\alpha+i \tau) d \tau}{z^{2}-(\alpha+i \tau)^{2}}=0 .
$$

Thus from (2.16) and (2.7) we arrive at the expansion (1.2) and complete the proof of Theorem 2.1.

COROLLARY 2.2. The Lebedev transformation (1.4) is a bounded operator from $\mathrm{H}_{2, A}$ into the space $L_{1}\left(\mathbb{R}_{+} ;\left|I_{\beta}(t)\right| t^{-1} d t\right)$, where $\operatorname{Re} \beta>0$.

Proof. In fact, by taking $\operatorname{Re} \beta>|\alpha|$ from estimates (2.1), (2.5) we find

$$
\|g\|_{L_{1}\left(\mathbb{R}_{+} ;\left|I_{\beta}(t)\right| t^{-1} d t\right)}=\int_{0}^{\infty}\left|I_{\beta}(t)\right| \frac{|g(t)|}{t} d t \leq \text { const. }\|f\|_{H_{2, A}} .
$$

We consider Lebedev's transformation (1.4) when $f$ belongs to $\hat{H}_{2, A}$ with the norm (1.7). The main result is established by the following.

THEOREM 2.3. Let an odd function $f \in \hat{H}_{2, A}, 0<A<1$. Then Lebedev's transform (1.4) belongs to $L_{2}\left(\mathbb{R}_{+} ; x^{-1} d x\right)$ and satisfies the following identity:

$$
\int_{0}^{\infty}|g(x)|^{2} \frac{d x}{x}=\int_{-\infty}^{+\infty} \frac{\operatorname{Re} z-i \tau}{\sin \pi(\operatorname{Re} z-i \tau)} \overline{f(\operatorname{Re} z+i \tau)} f(-\operatorname{Re} z+i \tau) d \tau .
$$

Moreover, for almost all $x>0, g(x)$ is defined by the formula

$$
g(x)=\frac{1}{\pi i} \frac{d}{d x} \int_{\alpha-i \infty}^{\alpha+i \infty} \mu \int_{0}^{x} K_{\mu}(y) f(\mu) d y d \mu, \quad|\alpha|<A .
$$

Finally, if $g \in L_{2}\left(\mathbb{R}_{+} ; t^{-1} d t\right) \cap L_{1}\left((0,1) ; t^{-A-1} d t\right)$, then the reciprocal inversion is of the form

$$
f(z)=-\frac{\sin \pi z}{\pi} \int_{0}^{\infty} K_{z}(t) \frac{g(t) d t}{t}, \quad|\operatorname{Re} z|<A .
$$

Proof. We prove that when $f \in H_{2, A}$, then the corresponding Lebedev transformation $g$ is a function from $L_{2}\left(\mathbb{R}_{+} ; x^{-1} d x\right)$. In order to proceed with this we show that for all $x>0$,

$$
\int_{\alpha-i \infty}^{\alpha+i \infty} \mu K_{\mu}(x) f(\mu) d \mu=\int_{-i \infty}^{i \infty} \mu K_{\mu}(x) f(\mu) d \mu .
$$

Then the right-hand side of (2.24) is the Kontorovich-Lebedev transform of if $(i \tau) \in$ $L_{2}(\mathbb{R}) \subset L_{2}(\mathbb{R} ; \tau d \tau / \sinh \pi \tau)$, which belongs to $L_{2}\left(\mathbb{R}_{+} ; x^{-1} d x\right)$ due to a Plancherel type theorem (see, e.g., [9, 10]).

So, appealing to representations (1.11) we find that the integrand in (2.24) is analytic by $\mu$ in the strip $|\operatorname{Re} \mu|<A$ for each $x>0$. Therefore by Cauchy's theorem we obtain

$$
\int_{C}^{C+1} d B\left[\int_{\alpha+i B}^{\alpha-i B}+\int_{\alpha-i B}^{-i B}+\int_{-i B}^{i B}+\int_{i B}^{\alpha+i B}\right] \mu K_{\mu}(x) f(\mu) d \mu=0 .
$$


Hence we treat each integral in (2.25) in a similar manner to that in the proof of Theorem 2.1 by using Schwarz's inequality and the above estimates for the last integral in (2.1). Then it is not difficult to establish the following relations:

$$
\begin{aligned}
& \lim _{C \rightarrow \infty} \int_{C}^{C+1} d B \int_{\alpha-i B}^{-i B} \mu K_{\mu}(x) f(\mu) d \mu=0, \\
& \lim _{C \rightarrow \infty} \int_{C}^{C+1} d B \int_{i B}^{\alpha+i B} \mu K_{\mu}(x) f(\mu) d \mu=0, \\
& \lim _{C \rightarrow \infty} \int_{C}^{C+1} d B \int_{\alpha+i B}^{\alpha-i B} \mu K_{\mu}(x) f(\mu) d \mu=\int_{\alpha+i \infty}^{\alpha-i \infty} \mu K_{\mu}(x) f(\mu) d \mu, \\
& \lim _{C \rightarrow \infty} \int_{C}^{C+1} d B \int_{-i B}^{+i B} \mu K_{\mu}(x) f(\mu) d \mu=\int_{-i \infty}^{i \infty} \mu K_{\mu}(x) f(\mu) d \mu,
\end{aligned}
$$

which lead to equality (2.24). Similarly it is not difficult to verify that for any $f \in H_{2, A}$, we can shift a contour of integration within the strip $|\operatorname{Re} \mu|<A$ for the integral involving the modified Bessel function (1.9). In particular, analogously to (2.25), we write

$$
\int_{C}^{C+1} d B\left[\int_{-\alpha+i B}^{-\alpha-i B}+\int_{-\alpha-i B}^{\alpha-i B}+\int_{\alpha-i B}^{\alpha+i B}+\int_{\alpha+i B}^{-\alpha+i B}\right] \frac{\mu}{\sin \pi \mu} I_{ \pm \mu}(x) f(\mu) d \mu=0 .
$$

When $C \rightarrow \infty$, we derive the equality

$$
\int_{\alpha-i \infty}^{\alpha+i \infty} \frac{\mu}{\sin \pi \mu} I_{ \pm \mu}(x) f(\mu) d \mu=\int_{-\alpha-i \infty}^{-\alpha+i \infty} \frac{\mu}{\sin \pi \mu} I_{ \pm \mu}(x) f(\mu) d \mu, \quad|\operatorname{Re} \mu|<A .
$$

Now we prove (2.21) for $f \in H_{2, A}$. We note that the case $\operatorname{Re} z=0$ corresponds to the Parseval equality for the Kontorovich-Lebedev transform [9, 10]. Hence assuming that $0<\operatorname{Re} z<A(-A<\operatorname{Re} z<0)$ by virtue of Theorem 2.1, we substitute in the right-hand side of (2.21) instead of $f(\operatorname{Re} z+i \tau)(f(-\operatorname{Re} z+i \tau))$ its value by the transformation (1.5). Then it becomes

$$
\begin{aligned}
& \int_{-\infty}^{+\infty} \frac{\operatorname{Re} z-i \tau}{\sin \pi(\operatorname{Re} z-i \tau)} \overline{f(\operatorname{Re} z+i \tau)} f(-\operatorname{Re} z+i \tau) d \tau \\
& \quad=\int_{-\infty}^{+\infty} \frac{\operatorname{Re} z-i \tau}{\sin \pi(\operatorname{Re} z-i \tau)} f(-\operatorname{Re} z+i \tau) \int_{0}^{\infty} \frac{I_{\operatorname{Re} z-i \tau}(t)}{t} \overline{g(t)} d t d \tau
\end{aligned}
$$

where $g(t)$ is defined by (1.4). We motivate the change of the order of integration in the right-hand side of (2.29) by Fubini's theorem via the estimate (see Corollary 2.2)

$$
\begin{aligned}
& \int_{-\infty}^{\infty}\left|\frac{\operatorname{Re} z-i \tau}{\sin \pi(\operatorname{Re} z-i \tau)} f(-\operatorname{Re} z+i \tau)\right| \int_{0}^{\infty} \frac{\left|I_{\operatorname{Re} z-i \tau}(t)\right|}{t}|g(t)| d t \\
& \quad \leq \text { const. }\left(\int_{-\infty}^{\infty}\left|\frac{\operatorname{Re} z-i \tau}{\sin \pi(\operatorname{Re} z-i \tau)}\right|^{2} d \tau\right)^{1 / 2}\|f\|_{H_{2, A}}^{2}<\infty, \quad 0<\operatorname{Re} z<A<1 .
\end{aligned}
$$

Inverting the order of integration we treat the inner integral by using relation (1.10). Hence taking into account the relations (2.28) and since $f(z)=-f(-z),|\operatorname{Re} z|<A$, 
we obtain

$$
\begin{aligned}
\int_{-\infty}^{\infty} & \frac{\operatorname{Re} z-i \tau}{\sin \pi(\operatorname{Re} z-i \tau)} I_{\operatorname{Re} z-i \tau}(t) f(-\operatorname{Re} z+i \tau) d \tau \\
& =\frac{1}{\pi i} \int_{\operatorname{Re} z-i \infty}^{\operatorname{Re} z+i \infty} \frac{\pi \bar{z}}{2 \sin \pi \bar{z}} I_{\bar{z}}(t)[f(-\bar{z})-f(\bar{z})] d z \\
& =\frac{1}{\pi i} \int_{\operatorname{Re} z-i \infty}^{\operatorname{Re} z+i \infty} \frac{\pi z}{2 \sin \pi z} I_{z}(t)[f(-z)-f(z)] d z \\
& =\frac{1}{\pi i} \int_{-\operatorname{Re} z-i \infty}^{-\operatorname{Re} z+i \infty} \frac{\pi z}{2 \sin \pi z} I_{-z}(t) f(z) d z-\frac{1}{\pi i} \int_{\operatorname{Re} z-i \infty}^{\operatorname{Re} z+i \infty} \frac{\pi z}{2 \sin \pi z} I_{z}(t) f(z) d z \\
& =\frac{1}{\pi i} \int_{\operatorname{Re} z-i \infty}^{\operatorname{Re} z+i \infty} \frac{\pi z}{2 \sin \pi z}\left[I_{-z}(t)-I_{z}(t)\right] f(z) d z \\
& =\frac{1}{\pi i} \int_{\operatorname{Re} z-i \infty}^{\operatorname{Re} z+i \infty} z K_{z}(t) f(z) d z=g(t)
\end{aligned}
$$

and therefore we arrive at the left-hand side of equality (2.21). Since the space $H_{2, A}$ is dense in $\hat{H}_{2, A}$, then for each $f \in \hat{H}_{2, A}$ we have $f(z)=\lim _{n \rightarrow \infty} f_{n}(z)$, where $f_{n} \in H_{2, A}$ and the latter limit is with respect to the norm (1.7). Furthermore, by a well-known relation for the gamma function (cf., [1]),

$$
\frac{\pi z}{\sin \pi z}=\Gamma(1+z) \Gamma(1-z)
$$

and invoking Schwarz's inequality we have from (2.21) correspondingly,

$$
\begin{aligned}
\int_{0}^{\infty}\left|g_{n}(x)-g_{m}(x)\right|^{2} \frac{d x}{x} \\
=\frac{1}{\pi}\left|\int_{\operatorname{Re} z-i \infty}^{\operatorname{Re} z+i \infty} \overline{\overline{\sin \pi z}} \overline{\left(f_{n}(z)-f_{m}(z)\right)}\left(f_{n}(-\bar{z})-f_{m}(-\bar{z})\right) d z\right| \\
\leq \frac{1}{\pi}\left(\int_{-\infty}^{\infty}|\Gamma(1+\operatorname{Re} z+i \tau)|^{2}\left|f_{n}(\operatorname{Re} z+i \tau)-f_{m}(\operatorname{Re} z+i \tau)\right|^{2} d \tau\right)^{1 / 2} \\
\quad \times\left(\int_{-\infty}^{\infty}|\Gamma(1-\operatorname{Re} z-i \tau)|^{2}\left|f_{n}(-\operatorname{Re} z+i \tau)-f_{m}(-\operatorname{Re} z+i \tau)\right|^{2} d \tau\right)^{1 / 2} \\
\leq \frac{1}{\pi}|| f_{n}-f_{m} \|_{\hat{H}_{2, A}}^{2} \rightarrow 0, \quad n, m \rightarrow \infty .
\end{aligned}
$$

Therefore $g_{n}(x)$ is a Cauchy sequence in the space $L_{2}\left(\mathbb{R}_{+} ; x^{-1} d x\right)$ and has a limit $g \in$ $L_{2}\left(\mathbb{R}_{+} ; x^{-1} d x\right)$, which we call Lebedev's transformation of $f \in \hat{H}_{2, A}$. We will show that for almost all $x>0$ the Lebedev transformation is defined by (2.22), which coincides, in turn, with (1.4) when $f \in H_{2, A}$. Indeed, integrating the equality

$$
g_{n}(t)=\frac{1}{\pi i} \int_{\alpha-i \infty}^{\alpha+i \infty} \mu K_{\mu}(t) f_{n}(\mu) d \mu,
$$

with respect to $t \in[0, x]$, we invert the order of integration in its right-hand side. This is motivated by estimates (2.1), (2.4) and by the convergence of the integral (see (2.5))

$$
\int_{0}^{x} t\left(K_{2(\alpha+1)}(2 t)+K_{2(\alpha-1)}(2 t)-2 K_{2 \alpha}(2 t)\right)^{1 / 2} d t<\infty, \quad|\alpha|<1 .
$$


Thus we obtain

$$
\int_{0}^{x} g_{n}(t) d t=\frac{1}{\pi i} \int_{\alpha-i \infty}^{\alpha+i \infty} \mu \int_{0}^{x} K_{\mu}(t) f_{n}(\mu) d t d \mu
$$

However, since

$$
\int_{0}^{x}\left|g_{n}(t)\right| d t \leq\left(\int_{0}^{x} t d t\right)^{1 / 2}\left\|g_{n}\right\|_{L_{2}\left(\mathbb{R}_{+} ; x^{-1} d x\right)}<\infty
$$

we have that

$$
\lim _{n \rightarrow \infty} \int_{0}^{x} g_{n}(t) d t=\int_{0}^{x} g(t) d t
$$

On the other hand, with Schwarz's inequality, the right-hand side of (2.36) is majorized by

$$
\begin{aligned}
& \frac{1}{\pi}\left|\int_{\alpha-i \infty}^{\alpha+i \infty} \mu \int_{0}^{x} K_{\mu}(t) f_{n}(\mu) d t d \mu\right| \\
& \quad \leq \frac{1}{\pi}\left(\int_{-\infty}^{\infty}\left|\frac{\alpha+i \tau}{\Gamma(1+\alpha+i \tau)}\right|^{2}\left|\int_{0}^{x} K_{\alpha+i \tau}(t) d t\right|^{2} d \tau\right)^{1 / 2}\left\|f_{n}\right\|_{\hat{H}_{2, A}} .
\end{aligned}
$$

Consequently, this is finite if the latter integral is convergent. Then we pass to the limit in (2.36) by $n \rightarrow \infty$ and, combining with (2.38), we derive the equality

$$
\int_{0}^{x} g(t) d t=\frac{1}{\pi i} \int_{\alpha-i \infty}^{\alpha+i \infty} \mu \int_{0}^{x} K_{\mu}(t) f(\mu) d t d \mu .
$$

Hence for almost all $x>0$ it leads to (2.22). Moreover, it coincides with (1.4) when $f \in H_{2, A}$, since in this case we may put the derivative inside the integral via its uniform convergence.

In order to prove that the integral in (2.39) is finite we use (1.10), relation (2.32), and the reduction formula for the gamma function $\Gamma(z+1)=z \Gamma(z)$ [1]. Then invoking Minkowski's inequality we easily find

$$
\begin{aligned}
\left(\int_{-\infty}^{\infty}\left|\frac{\alpha+i \tau}{\Gamma(1+\alpha+i \tau)}\right|^{2}\left|\int_{0}^{x} K_{\alpha+i \tau}(t) d t\right|^{2} d \tau\right)^{1 / 2} \\
=\frac{1}{2}\left(\int_{-\infty}^{\infty}\left|\frac{\alpha+i \tau}{\Gamma(1+\alpha+i \tau)}\right|^{2}\left|\frac{\pi}{\sin \pi(\alpha+i \tau)}\right|^{2}\left|\int_{0}^{x}\left[I_{-\alpha-i \tau}(t)-I_{\alpha+i \tau}(t)\right] d t\right|^{2} d \tau\right)^{1 / 2} \\
=\frac{1}{2}\left(\int_{-\infty}^{\infty}|\Gamma(1-\alpha+i \tau)|^{2}\left|\int_{0}^{x}\left[I_{-\alpha-i \tau}(t)-I_{\alpha+i \tau}(t)\right] d t\right|^{2} d \tau\right)^{1 / 2} \\
\leq \frac{1}{2}\left(\int_{-\infty}^{\infty}|\Gamma(1-\alpha+i \tau)|^{2}\left|\int_{0}^{x} I_{-\alpha-i \tau}(t) d t\right|^{2} d \tau\right)^{1 / 2} \\
\quad+\frac{1}{2}\left(\int_{-\infty}^{\infty}|\Gamma(1-\alpha+i \tau)|^{2}\left|\int_{0}^{x} I_{\alpha+i \tau}(t) d t\right|^{2} d \tau\right)^{1 / 2}
\end{aligned}
$$


Meanwhile, from (1.9) and the relation $\Gamma(k+1 \pm(\alpha+i \tau))=\Gamma(1 \pm(\alpha+i \tau))(1 \pm(\alpha+i \tau))_{k}$, we have, for each $x>0$,

$$
\begin{aligned}
& \left|\int_{0}^{x} I_{ \pm(\alpha+i \tau)}(t) d t\right| \\
& \quad=\frac{1}{|\Gamma(1 \pm(\alpha+i \tau))|}\left|\int_{0}^{x}\left(\frac{t^{ \pm(\alpha+i \tau)}}{2^{ \pm(\alpha+i \tau)}}+\sum_{k=1}^{\infty} \frac{t^{ \pm(\alpha+i \tau)+2 k}}{2^{ \pm(\alpha+i \tau)+2 k}(1 \pm(\alpha+i \tau))_{k} k !}\right) d t\right| \\
& \quad \leq \frac{1}{|\Gamma(1 \pm(\alpha+i \tau))|}\left|\int_{0}^{x} \frac{t^{ \pm(\alpha+i \tau)} d t}{2^{ \pm(\alpha+i \tau)} \mid}\right| \frac{1}{|\Gamma(1 \pm(\alpha+i \tau))|} \sum_{k=1}^{\infty} \frac{x^{ \pm \alpha+2 k+1}}{2^{ \pm \alpha+2 k}\left|(1 \pm(\alpha+i \tau))_{k}\right| k !} \\
& \quad=O\left(\frac{1}{|\tau \Gamma(1 \pm(\alpha+i \tau))|}\right), \quad|\tau| \longrightarrow \infty .
\end{aligned}
$$

Hence we use this estimate after splitting the last integrals in (2.41) with respect to $\tau$ on two integrals over $|\tau|<M$ and $|\tau| \geq M, M>0$. It results immediately in their convergence. Therefore the integral in (2.39) is finite.

Finally we prove the reciprocal formula (2.23). Indeed, for two different functions $f, f_{1} \in \hat{H}_{2, A}$ and the corresponding $g, g_{1} \in L_{2}\left(\mathbb{R}_{+} ; t^{-1} d t\right)$, we may write (2.21) in the form

$$
\int_{0}^{\infty} g(t) \overline{g_{1}(t)} \frac{d t}{t}=\int_{-\infty}^{\infty} \frac{\operatorname{Re} z-i \tau}{\sin \pi(\operatorname{Re} z-i \tau)} f(-\operatorname{Re} z+i \tau) \overline{f_{1}(\operatorname{Re} z+i \tau)} d \tau .
$$

Meantime, the theorem due to Paley and Wiener [6] says that the class $H_{2}(-A, A)$ of analytic functions in the strip $|\operatorname{Re} z| \leq A$ with the norm (1.3) over this strip coincides with the set of functions, which admit the representation

$$
F(z)=\frac{1}{\sqrt{2 \pi}} \int_{-\infty}^{\infty} e^{-z t} \varphi(t) d t, \quad-A<\operatorname{Re} z<A,
$$

where the integral is absolutely convergent and a measurable function $\varphi(t)$ is such that $\varphi \in L_{2}\left(\mathbb{R}_{-} ; e^{-2 A t} d t\right)$ and $\varphi \in L_{2}\left(\mathbb{R}_{+} ; e^{2 A t} d t\right)$. Hence by taking

$$
\varphi_{1}(t)= \begin{cases}1 & \text { if } t \in[0, x], \\ 0 & \text { if } t \in \mathbb{R} \backslash[0, x],\end{cases}
$$

we have correspondingly from (2.44) that for each $x>0, f_{1}(\operatorname{Re} z+i \tau)=(1 / \sqrt{2 \pi})((1-$ $\left.\left.e^{-(\operatorname{Re} z+i \tau) x}\right) /(\operatorname{Re} z+i \tau)\right) \in \hat{H}_{2, A}$. But the latter function is also from the space $H_{2}(-A, A)$. Therefore by (1.4) we find

$$
g_{1}(t, x)=\frac{1}{\pi \sqrt{2 \pi}} \int_{-\infty}^{\infty} K_{\operatorname{Re} z+i \tau}(t)\left[1-e^{-(\operatorname{Re} z+i \tau) x}\right] d \tau .
$$

We may calculate explicitly the value of the function $g_{1}(t, x)$ by using $[7$, relation (2.16.48.19)] and the Cauchy theorem. Then we obtain $g_{1}(t, x)=\left(e^{-t}-e^{-t \cosh x}\right) / \sqrt{2 \pi}$. Further, from (1.7) and (2.32), we see that $(z / \sin \pi z) f(z) \in H_{2}(-A, A), 0<A<1$. 
Consequently, in view of (2.44) there exists a measurable function $\varphi(t)$ such that

$$
\frac{z}{\sin \pi z} f(z)=\frac{1}{\sqrt{2 \pi}} \int_{-\infty}^{\infty} e^{-z t} \varphi(t) d t
$$

where $\operatorname{Re} z=\alpha,|\alpha|<A, \varphi \in L_{2}\left(\mathbb{R}_{-} ; e^{-2 A t} d t\right)$, and $\varphi \in L_{2}\left(\mathbb{R}_{+} ; e^{2 A t} d t\right)$. Hence by the Parseval equality for the Fourier transform, the right-hand side of (2.43) may be written as

$$
\int_{-\infty}^{\infty} \frac{\alpha-i \tau}{\sin \pi(\alpha-i \tau)} f(-\alpha+i \tau) \overline{f_{1}(\alpha+i \tau)} d \tau=\int_{-\infty}^{\infty} \varphi(t) \overline{\varphi_{1}(t)} d t=\int_{0}^{x} \varphi(t) d t .
$$

Combining with (2.46) and the left-hand side of (2.43), we derive

$$
\int_{0}^{x} \varphi(t) d t=\frac{1}{\sqrt{2 \pi}} \int_{0}^{\infty} g(t)\left[e^{-t}-e^{-t \cosh x}\right] \frac{d t}{t}
$$

or, for almost all $x>0$,

$$
\varphi(x)=\frac{1}{\sqrt{2 \pi}} \frac{d}{d x} \int_{0}^{\infty} g(t)\left[e^{-t}-e^{-t \cosh x}\right] \frac{d t}{t} .
$$

However, we may differentiate through the integral sign in (2.50) and write it as follows:

$$
\varphi(x)=\frac{\sinh x}{\sqrt{2 \pi}} \int_{0}^{\infty} g(t) e^{-t \cosh x} d t
$$

In fact, this is motivated by the uniform convergence of the integral (2.51) on $x \geq 0$ since, via Schwarz's inequality, we obtain

$$
\begin{aligned}
\sinh x\left|\int_{0}^{\infty} g(t) e^{-t \cosh x} d t\right| & \leq \sinh x\|g\|_{L_{2}\left(\mathbb{R}_{+} ; t^{-1} d t\right)}\left(\int_{0}^{\infty} t e^{-2 t \cosh x} d t\right)^{1 / 2} \\
& =\frac{\tanh x}{2}\|g\|_{L_{2}\left(\mathbb{R}_{+} ; t^{-1} d t\right)} \leq \frac{1}{2}\|g\|_{L_{2}\left(\mathbb{R}_{+} ; t^{-1} d t\right) .}
\end{aligned}
$$

Hence substituting (2.51) into (2.47) we find

$$
\frac{z}{\sin \pi z} f(z)=\frac{1}{2 \pi} \int_{-\infty}^{\infty} e^{-z y} \sinh y \int_{0}^{\infty} g(t) e^{-t \cosh y} d y d t
$$

If we invert formally the order of integration in (2.53), then, calculating the integral with respect to $y$ by using (1.11), (1.12), we arrive at the inversion formula (2.23). In order to complete the proof of the theorem we show that this interchange is indeed possible due to Fubini's theorem under condition $g \in L_{2}\left(\mathbb{R}_{+} ; t^{-1} d t\right) \cap L_{1}\left((0,1) ; t^{-A-1} d t\right)$. In fact, 
by the Schwarz inequality we have

$$
\begin{aligned}
\mid \int_{-\infty}^{\infty} & e^{-(\alpha+i \tau) y} \sinh y \int_{0}^{\infty} g(t) e^{-t \cosh y} d y d t \mid \\
\leq & \left(\int_{-\infty}^{\infty} e^{2 A y} \sinh ^{2} y\left|\int_{0}^{\infty} g(t) e^{-t \cosh y} d t\right|^{2} d y\right)^{1 / 2} \\
& \times\left[\left(\int_{0}^{\infty} e^{-2(A+\alpha) y} d y\right)^{1 / 2}+\left(\int_{-\infty}^{0} e^{2(A-\alpha) y} d y\right)^{1 / 2}\right] \\
= & {\left[\frac{1}{\sqrt{2(A+\alpha)}}+\frac{1}{\sqrt{2(A-\alpha)}}\right] } \\
& \times\left(\int_{-\infty}^{\infty} e^{2 A y} \sinh ^{2} y\left|\int_{0}^{\infty} g(t) e^{-t \cosh y} d t\right|^{2} d y\right)^{1 / 2}, \quad|\alpha|<A .
\end{aligned}
$$

Meanwhile, employing the generalized Minkowski inequality, we derive

$$
\begin{aligned}
& \left(\int_{-\infty}^{\infty} e^{2 A y} \sinh ^{2} y\left|\int_{0}^{\infty} g(t) e^{-t \cosh y} d t\right|^{2} d y\right)^{1 / 2} \\
& \quad \leq \int_{0}^{\infty}|g(t)| \times\left(\int_{-\infty}^{\infty} e^{2 A y} e^{-2 t \cosh y} \sinh ^{2} y d y\right)^{1 / 2} d t .
\end{aligned}
$$

Integrating by parts in the latter integral and using again (1.11), (1.12), we obtain

$$
\begin{aligned}
\left(\int_{-\infty}^{\infty}\right. & \left.e^{2 A y} e^{-2 t \cosh y} \sinh ^{2} y d y\right)^{1 / 2} \\
& =\sqrt{2}\left(\int_{0}^{\infty} e^{-2 t \cosh y} \cosh 2 A y \sinh ^{2} y d y\right)^{1 / 2} \\
& =\left(\frac{1}{t} \int_{0}^{\infty} e^{-2 t \cosh y}[\cosh 2 A y \cosh y+2 A \sinh 2 A y \sinh y] d y\right)^{1 / 2} \\
& =\left(\frac{1}{2 t}\left[K_{2 A+1}(2 t)+K_{2 A-1}(2 t)\right]+\frac{2 A^{2}}{t^{2}} K_{2 A}(2 t)\right)^{1 / 2}
\end{aligned}
$$

Consequently, invoking (1.15), (1.17), we see that

$$
\begin{aligned}
& \left(\frac{1}{2 t}\left[K_{2 A+1}(2 t)+K_{2 A-1}(2 t)\right]+\frac{2 A^{2}}{t^{2}} K_{2 A}(2 t)\right)^{1 / 2}=O\left(t^{-A-1}\right), \quad t \rightarrow 0, \\
& \left(\frac{1}{2 t}\left[K_{2 A+1}(2 t)+K_{2 A-1}(2 t)\right]+\frac{2 A^{2}}{t^{2}} K_{2 A}(2 t)\right)^{1 / 2}=O\left(e^{-t} t^{-3 / 4}\right), \quad t \rightarrow+\infty
\end{aligned}
$$

and combining with (2.55) we arrive at the estimate

$$
\begin{aligned}
& \left|\int_{-\infty}^{\infty} e^{-(\alpha+i \tau) y} \sinh y \int_{0}^{\infty} g(t) e^{-t \cosh y} d y d t\right| \\
& \quad \leq \text { const. } \int_{0}^{1}|g(t)| t^{-A-1} d t+\text { const. } \int_{1}^{\infty}|g(t)|^{2} \frac{d t}{t}<\infty .
\end{aligned}
$$

This ends the proof of Theorem 2.3. 
ACKNOWLEDGMENTS. The present investigation was supported, in part, by the Centre of Mathematics of the University of Porto. The author is indebted to the referee for the valuable comments and remarks, which helped improve the presentation of the paper.

\section{REFERENCES}

[1] A. Erdélyi, W. Magnus, F. Oberhettinger, and F. G. Tricomi, Higher Transcendental Functions. I, McGraw-Hill Book Company, New York, 1953.

[2] , Higher Transcendental Functions. II, McGraw-Hill Book Company, New York, 1953.

[3] K. Hoffman, Banach Spaces of Analytic Functions, Prentice-Hall Series in Modern Analysis, Prentice-Hall, New Jersey, 1962.

[4] N. N. Lebedev, On the representation of arbitrary function by an integral involving Macdonald functions of complex order, Doklady Akad. Nauk SSSR (N. S.) 58 (1947), no. 6, 1007-1010 (Russian).

[5] Y. L. Luke, The Special Functions and Their Approximations, Vol. I, Mathematics in Science and Engineering, vol. 53, Academic Press, New York, 1969.

[6] R. Paley and N. Wiener, Fourier Transforms in the Complex Domain, American Mathematical Society, Rhode Island, 1934.

[7] A. P. Prudnikov, Yu. A. Brychkov, and O. I. Marichev, Integrals and Series. Vol. 2. Special Functions, Gordon and Breach Science Publishers, New York, 1986.

[8] E. C. Titchmarsh, Indroduction to the Theory of Fourier Integrals, Clarendon Press, Oxford, 1937.

[9] S. B. Yakubovich, Index Transforms, World Scientific Publishing, New Jersey, 1996.

[10] - On the index integral transformation with Nicholson's function as the kernel, J. Math. Anal. Appl. 269 (2002), no. 2, 689-701.

Semyon B. Yakubovich: Department of Pure Mathematics, Faculty of Science, University of Porto, 687 Campo Alegre Street, 4169-007 Porto, Portugal

E-mail address: syakubov@fc.up.pt 


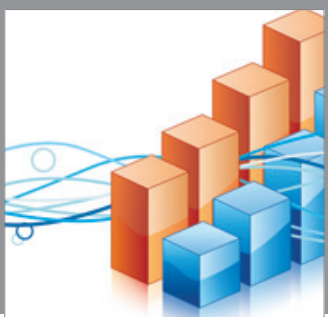

Advances in

Operations Research

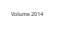

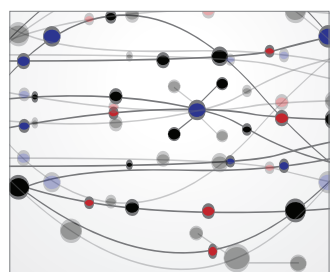

\section{The Scientific} World Journal
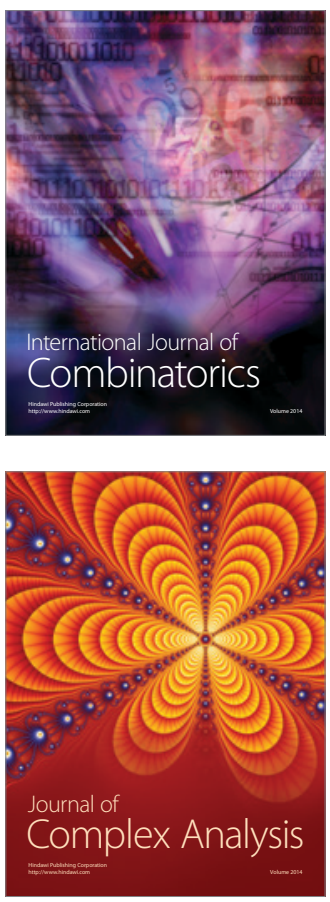

International Journal of

Mathematics and

Mathematical

Sciences
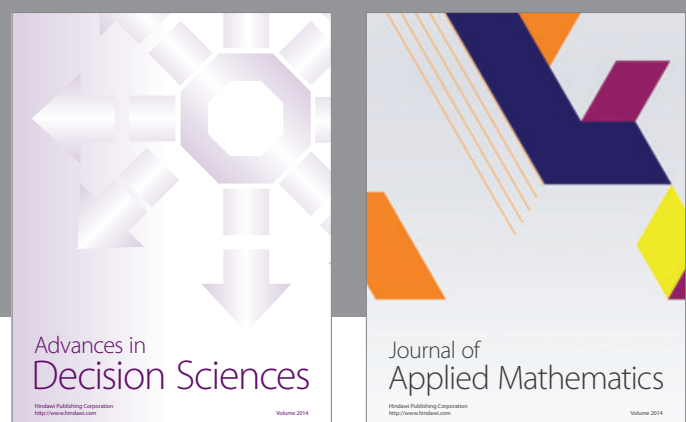

Journal of

Applied Mathematics
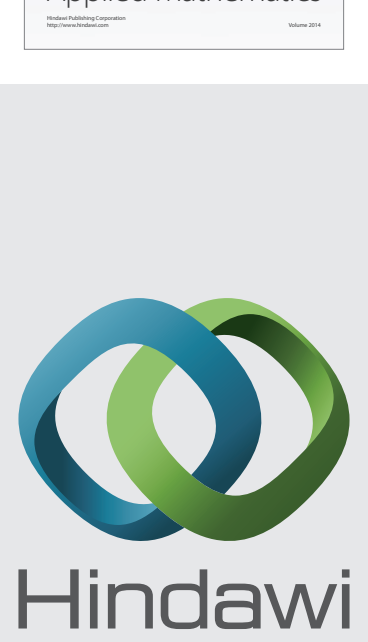

Submit your manuscripts at http://www.hindawi.com
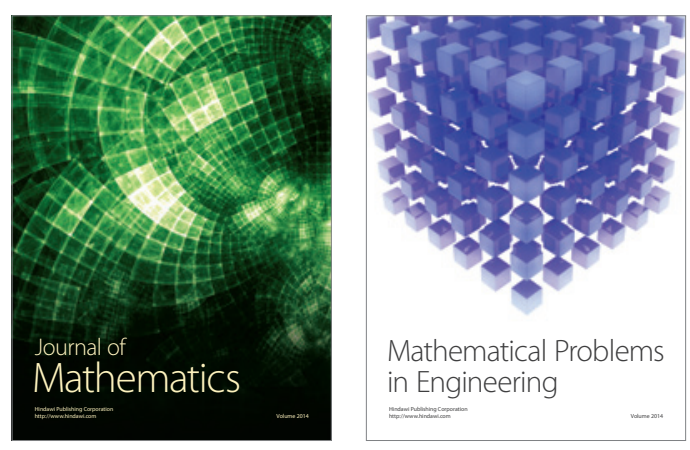

Mathematical Problems in Engineering
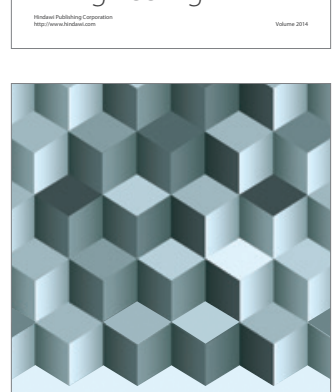

Journal of

Function Spaces
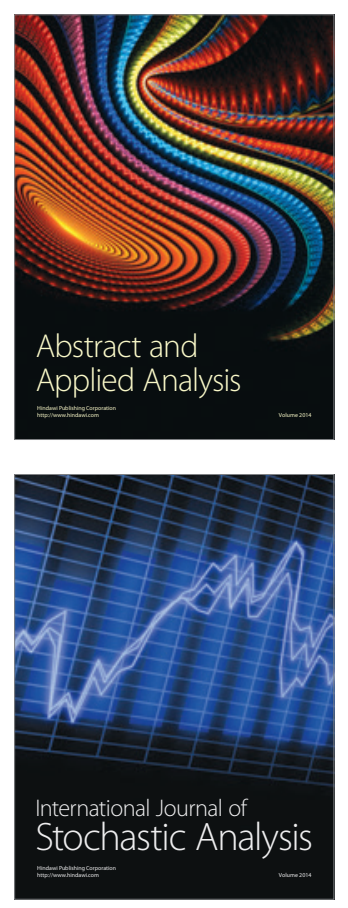

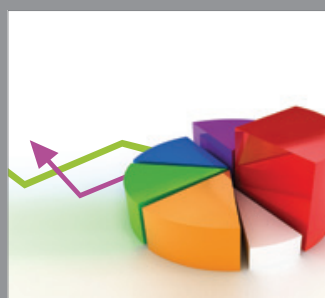

ournal of

Probability and Statistics

Promensencen
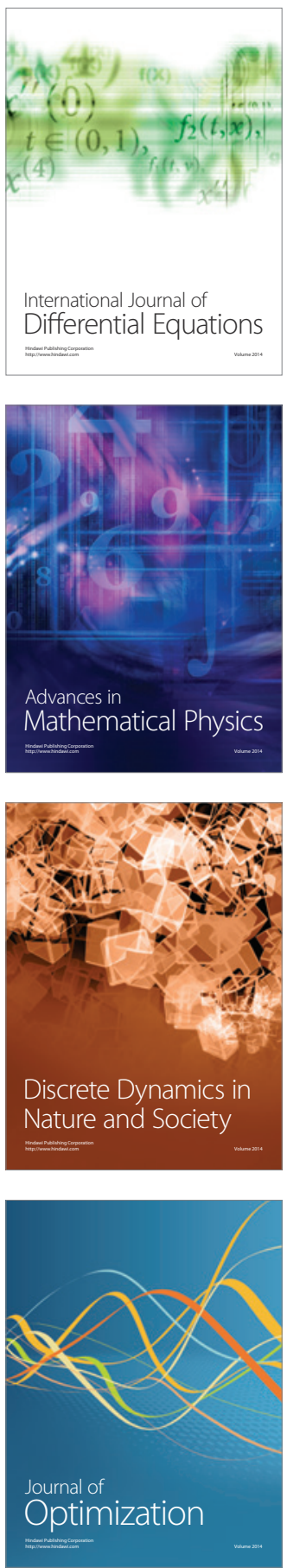